\title{
Efficacy of surgical treatment in the management of idiopathic granulomatous mastitis: an institutional experience in Sri Lanka
}

\author{
Ranali Perera ${ }^{1}$, Chatura Ratnasooriya ${ }^{1}$, Naomal Perera ${ }^{1}$, Nishani Fernando $^{1}$, Bimalka Senevirathne $^{2}$ \\ ${ }^{1}$ Department of Oncological Surgery, Lanka Hospitals, Colombo, Sri Lanka \\ ${ }^{2}$ Department of Pathology, Faculty of Medical Sciences, University of Sri Jayewardenepura, Sri Lanka
}

Keywords: Idiopathic granulomatous mastitis (IGM); benign breast disease; breast lumpectomy; chronic inflammation

\begin{abstract}
\section{Introduction}

Idiopathic Granulomatous Mastitis (IGM) is a rare, benign, chronic inflammatory breast disease which can mimic a carcinoma both clinically and radiologically. This study aims to establish the efficacy of surgical treatment in the management of IGM.
\end{abstract}

\section{Methods}

We have retrospectively analysed 25 patients diagnosed with IGM between 2012-2019 who were managed surgically with wide local excision (WLE). Ultrasound scans (USS) and fine-needle aspiration cytology (FNAC) were performed in all patients to confirm mastitis and to exclude malignancy. IGM was diagnosed by excisional biopsy. WLE was performed on all patients and were followed up to an average of 37 months. (range 3- 83 months, median 41.5 months)

\section{Results}

None of the patients showed recurrence of the disease during the period of follow-up. Post-operative complications observed included oozing from the surgical site $(8 \%)$, wound site infection (4\%), and minor skin necrosis (4\%).

\section{Conclusion}

In the past, IGM has been managed in different ways ranging from observation, steroids, incision and drainage, with variable recurrence rates. As there is no accepted guideline up to date regarding the most effective treatment for IGM, we'd like to propose a surgical treatment with WLE as the treatment of choice due to the high rate of success and low rate of recurrence shown by our institutional experience.

Correspondence: Naomal Perera

E-mail:dr.naomal@gmail.com

(iD)https://orcid.org/0000-0002-4101-9010

Received: 07-11-2020 Accepted: 16-12-2020

DOI: http://doi.org/10.4038/sljs.v38i3.8760

\section{Introduction}

Granulomatous mastitis is a rare benign breast disease that is characterized by non-caseating granulomatous inflammation of the perilobular region. It can be divided into two entities based on the presence or absence of a specific aetiology [1]. The common causes for granulomatous inflammation of the breast are infections (a known pathogen being Corynebacterium), trauma, and disease conditions like TB, sarcoidosis or autoimmune diseases [2-5].

Idiopathic granulomatous mastitis (IGM) as defined by Kessler and Wolloch in 1972 is a disease of unknown aetiology [6]. Various studies have sought to confirm the association of this condition with factors such as hyperprolactinaemia, breastfeeding, smoking, use of the oral contraceptive pill (OCP) and disease conditions like DM and autoimmune diseases $[1,3,5-7]$. It is the more common type of granulomatous mastitis, seen commonly in parous women of the reproductive age group, the mean age of presentation ranging from 28.4 to 37.75 years according to our literature review [7 -13].

It presents mainly as a painful lump, with the presence of inflammation, abscesses, sinuses or fistulae formation at times and often leads to a diagnostic dilemma [8, 12, 14]. IGM is commonly mistaken as breast carcinoma leading to unnecessary mastectomies or treated with incision and drainage following a wrongful diagnosis of breast abscess $[10,12,13,15]$.

Biopsy of IGM has revealed the presence of non-caseating granulomas, consisting of epithelioid cells, Langerhan type giant cells, lymphocytes and plasma cells $[10,11]$. Biopsy and histopathological review can help exclude other mimics namely $\mathrm{TB}$, breast carcinoma and abscess [4, 10, 13]. The presence of granulomatous reactions on biopsy has led to the improper use of antituberculous therapy following suspicion of tuberculous mastitis [10,16]. Exclusion of other diagnoses using laboratory, radiological and histopathological investigations is of vital importance in the accurate diagnosis of the condition.

Through time, it has been managed in numerous ways ranging from observation to steroids to mastectomy $[12,17,18]$. This 
study was done to assess the effectiveness of surgical management for IGM.

\section{Method}

Twenty-five consecutive patients with IGM diagnosed by ultrasound and FNAC, who visited the breast clinic at a private institution in Sri Lanka, were studied over 7 years from 2012 and retrospective analysis of data was done. The patients were assessed with regards to parity, breastfeeding, post lactation secretions, lactational mastitis and also for the association with potential risk factors; oral contraceptive pill (OCP) use, history of smoking, history of breast disease or surgery and prior diagnoses of other diseases such as tuberculosis (TB), sarcoidosis, diabetes mellitus and autoimmune diseases. An ultrasound scan (USS) was done preoperatively confirming mastitis with suppuration in all patients. Fine needle aspiration cytology (FNAC) confirmed the presence of acute/chronic inflammatory cells in all patients without any atypical or malignant cells. Following surgical excision of the mass, all cases were diagnosed as IGM histopathologically. Depending on the size of the defect, a rotational flap was used to fill the cavity. The fluid found within cavities; sinuses were taken for aerobic culture during surgery. All participants were followed up annually at the clinic for a period ranging from 3 months to 83 months with a median of 41.5 months.

\section{Results}

The study population had a mean age of 33.96 years, with the youngest aged 25 and the oldest aged 45 . All participants were parous women with a minimum parity of one and a maximum of three. All women had breastfed after each pregnancy.

All participants presented with a painful unilateral mass. The skin was visibly inflamed in $64 \%(n=16)$ of the study population while $60 \%(n=15)$ had sinuses with $50 \%$ of them having a serous discharge. $40 \%(n=10)$ had nipple involvement and only 1 participant had involvement of the axilla. Only 2 participants complained of fever ( $8 \%$ ).

Out of all patients studied, 2 had a history of OCP use (8\%) but none were smokers. One patient was a non-insulin-dependent diabetic $(4 \%)$ but none had a history of TB, sarcoidosis or autoimmune diseases. One participant $(4 \%)$ had a history of contralateral breast IGM and had undergone incision and drainage followed by wide local excision.

$56 \%(n=14)$ of participants has breastfed once before disease onset, while $36 \%(n=9)$ has breastfed twice and $8 \%(n=2)$ thrice. Majority of the participants has breastfed for 1-3 years with 4 patients $(16 \%)$ feeding for less than 6 months. Only 5 of those in the study population (20\%) has fed for more than 3 years.
In the pregnancy preceding presentation, 2 participants $(8 \%)$ developed mastitis on affected side progressing to lactational mastitis. 3 participants (12\%) developed nipple infection during the breastfeeding period preceding presentation.

Majority of the participants claimed not to have had any issues with milk production and $76 \%$ did not complain of secretion post lactation. Out of the 6 participants who complained of secretion post lactation, only 1 had up to 1 year, the rest resolving by 3 months.

Eight participants (32\%) had undergone incision and drainage prior to presentation to study centre (4 participants once, 2 participants twice, and 2 thrice).

During our study, the biopsy was performed for all participants following surgery. 96\% $(n=24)$ had epithelioid histiocytes, 88\% $(n=22)$ had giant cells, 60\% $(n=15)$ had abscess formation, $32 \%(\mathrm{n}=8)$ had duct ectasia and none had tumour cells or caseating necrosis. In all participants, the histological diagnosis was granulomatous mastitis without associated with other pathologies.

This diagnosis further collaborated with the culture reports. The aerobic culture was performed for all, out of which 100\% $(n=25)$ had negative cultures. Ziehl-Neelsen staining was done in all samples to exclude tuberculous mastitis.

All patients were managed surgically with WLE, and with flap closure in $60 \%(n=15)$. Majority of them had local rotational or advancement flaps using the glandular breast tissue $(n=12)$. Larger defects (greater than $5 \mathrm{~cm}$ ) were closed with either a mini latissimus dorsi flap ( $\mathrm{n}=1)$, a latissimus dorsi muscle flap $(n=1)$ or a latissimus dorsi myocutaneous flap $(n=1)$. The skin was included in the WLE of patients who displayed skin involvement with sinuses or inflammation.

None showed recurrence of the disease following surgery during the follow-up period. The complications observed were oozing from the surgical site, wound site infection and minor wound edge skin necrosis in $2(8 \%), 1(4 \%)$ and $1(4 \%)$ participants respectively.

\section{Discussion}

IGM is a rare disease presenting a diagnostic dilemma, but with proper clinical and laboratory assessment, an accurate diagnosis can be arrived at, with the exclusion of other disease mimics.

Diagnosis, however, is not the only area that raises a concern. This benign breast disease has and is being managed using different therapeutic approaches and is yet to have a standardized protocol [18]. Amongst the management options available are steroid use with or without methotrexate and other immunosuppressants, and the surgical treatment 
consisting of incision and drainage, wide local excision or mastectomy $[5,9,14,17]$.

Various studies conducted on IGM support surgery as a better therapeutic option. One study concluded that out of the 8 participants treated using wide local excision, only 2 had recurrences (25\% recurrence rate) in comparison to $50 \%$ recurrence rate when treated with incision and drainage and $50 \%$ recurrence rate for those treated with steroids [8]. Wilson JP et al analysed 116 cases of IGM and drew the following conclusions. $56 \%$ success rate with observation alone, $42 \%$ success rate with steroid therapy, $79 \%$ with partial mastectomy and a $100 \%$ success rate with mastectomy supporting surgery as a better modality of treatment [18].

A study done by Ozturk E et al showed zero recurrence rate for those managed with wide local excision (11 patients) and only one recurrence out of the subjects treated with incisional biopsy and medical management, who was then subsequently treated with abscess drainage and wide local excision [9].

The conclusions of these studies are following the results of our study.

All patients in our study were managed surgically with wide local excision.

Prior to the WLE that was done in our study, 8 had undergone incision and drainage, 2 of them twice, and 2 of them having undergone drainage thrice. The condition did not resolve in any of them following the procedure and resolved only after excision was performed through this study. It is a clear indication that IGM should not be managed as an abscess alone with incision and drainage, but with proper wide local excision to completely excise the inflammatory tissue that results in this condition, including the affected skin.

It is the authors' recommendation to use wide local excision with flap closure if a patient presents with a lump size greater than $5 \mathrm{~cm}$ in diameter or if there is the involvement of the skin. In our study population, all participants with a lump size greater than $5 \mathrm{~cm}$ underwent surgical excision with flap closure and had zero recurrence rates and zero complications during the subsequent follow-up period. Minor complications were noted only when WLE was done without utilizing a flap to close the defect, though they were self-limited. With a $100 \%$ success rate with wide excision, mastectomy becomes redundant and unnecessary in the management.

It is our observation that IGM requires complete excision of the inflammatory tissue. Failure to do so can result in a recurrence or poor resolution of the symptoms. Whether the inflammation occurs due to an immunological reaction to inspissated milk within the duct system has also been queried in the past [15]. The hypothesis of an immunological reaction to inspissated milk supports our surgical principle of wider excision of the affected breast tissues in IGM than in the performance of an incision and drainage. Moreover, it was noted that all participants in our study showed an abrupt cessation of lactation, and further immunological studies are warranted to confirm such a postulation.

\section{Conclusion}

IGM is a chronic inflammatory disease of the perilobular region of the breast tissue, which often leads to uncertainty during diagnosis and treatment. It requires the complete excision of inflammatory tissue for resolution. Our study concludes that wide local excision is an appropriate therapeutic option for successful management. Closure with flaps is preferred for masses greater than $5 \mathrm{~cm}$ and those involving the skin for complete resolution.

All authors disclose no conflict of interest. The study was conducted in accordance with the ethical standards of the relevant institutional or national ethics committee and the Helsinki Declaration of 1975, as revised in 2000 .

\section{References}

1. Altintoprak F, Kivilcim T, Ozkan OV. Aetiology of idiopathic granulomatous mastitis. World J Clin Cases. 2014 Dec 16;2(12):852-8. https://doi.org/10.12998/wjcc.v2.i12.852

2. Taylor GB, Paviour SD, Musaad S, et al. A clinicopathological review of 34 cases of inflammatory breast disease showing an association between corynebacteria infection and granulomatous mastitis. Pathology. 2003 Apr;35(2):109-19. https://doi.org/10.1097/01268031-200335020-00003

3. Cserni G, Szajki K. Granulomatous lobular mastitis following drug-induced galactorrhea and blunt trauma. Breast J. 1999 Nov;5(6):398-403. https://doi.org/10.1046/j.15244741.1999.97040.x

4. Elsiddig K E, Khalil E A, Elhag I A, et al. Granulomatous mammary disease: ten years' experience with fine needle aspiration cytology. Int J Tuberc Lung Dis. 2003 Apr;7(4):365-9. https://pubmed.ncbi.nlm.nih.gov/12729342/

5. Diesing D, Axt-Fliedner R, Hornung D, et al. Granulomatous mastitis. Arch Gynecol Obstet. 2004 May;269(4):233-6. https://doi.org/10.1007/s00404-003-0561-2

6. Kessler E, Wolloch Y. Granulomatous mastitis: a lesion clinically simulating carcinoma. Am J Clin Pathol. 1972 Dec;58(6):642-6. https://doi.org/10.1093/ajcp/58.6.642

7. Al-Khaffaf B, Knox F, Bundred NJ. Idiopathic granulomatous mastitis: a 25-year experience. J Am Coll Surg. 2008 Feb;206(2):269-73. https://doi.org/10.1016/j.jamcollsurg.2007.07.041

8. Subramanyam SG, Raja H, Gayatri R, et al. Idiopathic granulomatous mastitis - diagnostic and therapeutic dilemma. Int J Sci Study. 2018;6(7):51-55. https://www.ijsssn.com/uploads/2/0/1/5/20153321/10_ijss_oct_oa10__2018.pdf 
9. Ozturk E, Akin M, Can MF, et al. Idiopathic granulomatous mastitis. Saudi Med J. 2009 Jan;30(1):45-9.

https://pubmed.ncbi.nlm.nih.gov/19139772/

10. Akyildiz EU, Aydogan F, Ilvan S, et al. Idiopathic granulomatous mastitis. Eur J Breast Health. 2010;6(1):5-8. https://www.eurjbreasthealth.com/en/idiopathicgranulomatous-mastitis-13179

11.Going JJ, Anderson TJ, Wilkinson S, et al. Granulomatous lobular mastitis. J Clin Pathol. 1987 May;40(5):535-40. https://doi.org/10.1136/jcp.40.5.535

12.Thrush S, Dixon JM. Benign breast disease. In: Nixon JM, ed. A companion to specialist surgical practice: Breast surgery. 3rd ed. Elsevier Limited; 2006. https://doi.org/10.1002/bjs.5560

13.Bani-Hani KE, Yaghan RJ, Matalka II, et al. Idiopathic granulomatous mastitis: time to avoid unnecessary mastectomies. Breast J. 2004 Jul-Aug;10(4):318-22.

https://doi.org/10.1111/j.1075-122x.2004.21336.x

14.Aldaqal SM. Idiopathic granulomatous mastitis. Clinical presentation, radiological features and treatment. Saudi Med J. 2004 Dec;25(12):1884-7.

https://pubmed.ncbi.nlm.nih.gov/15711659/
15.Brown KL, Tang PH. Postlactational tumoral granulomatous mastitis: a localized immune phenomenon. Am J Surg. 1979 Aug;138(2):326-9. https://doi.org/10.1016/0002-9610(79) 90397-0

16.Agarwal C, Singh K, Pujani M, et al. Are all granulomatous mastitis cases tuberculous?: A study on the role of cytology in evaluation of granulomatous mastitis. Turk Patoloji Derg. 2019;35(2):128-133.

https://doi.org/10.5146/tjpath.2018.01442

17.Bouton ME, Jayaram L, O'Neill PJ, et al. Management of idiopathic granulomatous mastitis with observation. Am J Surg. 2015 Aug;210(2):258-62.

https://doi.org/10.1016/j.amjsurg.2014.08.044

18.Wilson JP, Massoll N, Marshall J, et al. Idiopathic granulomatous mastitis: in search of a therapeutic paradigm. Am Surg. 2007 Aug;73(8):798-802.

https://doi.org/10.1177/000313480707300813 